\title{
DIEULAFOY LESION AS A SOURCE OF BLEEDING: A REPORT OF TWO CLINICAL CASES
}

\section{Goran Sarafiloski, Mimi R. Marinova, Pencho T. Tonchev ${ }^{1}$}

Department of Gastroenterology, Medical University - Pleven

${ }^{1}$ Department of Surgery, Medical University - Pleven

\author{
Corresponding Author: \\ Goran Sarafiloski \\ Department of Gastroenterology, \\ Medical University - Pleven \\ 1, St. Kl. Ohridski Str. \\ Pleven, 5800 \\ Bulgaria \\ e-mail:dr_gorann@yahoo.com
}

Received: April 27, 2021

Revision received: May 11, 2021

Accepted: July 08, 2021

\section{Summary}

Dieulafoy's lesion is a rare source of gastrointestinal bleeding. It can be found anywhere in the gastrointestinal tract, but is most commonly seen in the proximal third of the stomach. It is a submucosal artery, adjacent to the mucosal surface, with no evidence of superficial ulcers. Lesions are routinely diagnosed and treated endoscopically. Their frequency is currently not well known, because as Dieulafoy lesions can be easily missed during endoscopy, and bleeding lesions are sometimes misidentified.In this article, we describe two cases of bleeding from the upper gastrointestinal tract, manifested by hematemesis and melena. Bleeding was successfully managed endoscopically by means of injection of adrenaline solution and application of an endoscopic hemoclip.

Keywords: Dieulafoy lesion, hemorrhage, hematemesis, endoscopic hemostasis, hemoclips

\section{Introduction}

In this article, we present two patients with acute upper gastrointestinal bleeding, manifested by hematemesis and melena, which underwent urgent diagnostic and therapeutic endoscopy. They were found to have acute bleeding from a Dieulafoy lesion.

Dieulafoy's lesion is a relatively rare but potentially life-threatening cause of gastrointestinal bleeding [1]. Its incidence amounts to $1-2 \%$ of all cases of acute gastrointestinal bleeding [2,3]. Before the introduction of endoscopy, the diagnosis was rarely made before surgery, and, in some cases, it was missed even during it. Mortality had reached $80 \%$. With the introduction of endoscopic and radiological techniques for diagnosis and treatment, mortality dropped to $13 \%$ [4].

It was first observed by Gallard in 1884 but was described in 1898 by Georges Dieulafoy, a French surgeon. He reported fatal gastric bleeding in three young men. Dieulafoy believed that this lesion was an early stage of peptic ulcer and called it exulceratio simplex [2,5].

Dieulafoy's lesion is a vascular abnormality localized in the submucosa. Histologically, it is a normal submucosal arterial blood vessel with an unusually large diameter of 1-3 $\mathrm{mm}$. The affected vessel has a curved course within the submucosa. The lesion usually comes 
out through a small mucosal defect ranging from 2-5 mm, which has fibrinoid necrosis at its base. It is located next to the mucosal surface and is subject to injury. The vascular abnormality erodes the proper epithelium and gets injured. The lesion is usually single and isolated. The presence of multiple lesions is extremely rare $[2,6]$.

The exact mechanism of rupture of the blood vessel and subsequent massive hemorrhage are not fully understood. One theory suggests that pulsations in the great submucosal blood vessel lead to disruption of the integrity of the integumentary epithelium, followed by local ischemia, erosion, and rupture. Another theory suggests that atrophy of the gastric mucosa promotes arterial thrombosis, leading to necrosis and hemorrhage. Old age and ischemic changes occurring in the gastric mucosa in cardiovascular disease further contribute to the occurrence of bleeding. Histologically, it has been proved that the artery wall has a normal architecture without signs of vasculitis, atherosclerosis or aneurysm formation. The hemorrhage is massive because the ruptured artery is usually large [7,8].

Dieulafoy's lesion is most often localized in the stomach. The defect of the mucosa is most often found in its proximal part, with a small curvature. About $80 \%$ to $95 \%$ of the lesions are located distally, $6 \mathrm{~cm}$ from the gastro-oesophageal ligament. This is due to the blood supply to the lesser curvature of the stomach, which is carried out directly by the branches of the left gastric artery. It can also be found in the duodenum $(14 \%)$, colon $(5 \%)$, surgical anastomoses $(5 \%)$, jejunum (1\%), and esophagus (1\%) [9].

The etiology is not fully understood. The finding is found twice as often in men, in middle age - in the fifth decade of life, and in older people. No inheritance is raised $[3,10]$. Risk factors include various comorbidities cardiopulmonary dysfunction and chronic renal failure and use of nonsteroidal anti-inflammatory drugs (NSAIDs) and aspirin [11].

Clinically, Dieulafoy's lesion presents as an acute massive hemorrhage with haematemesis and, less commonly, melena. Epigastric pain is not the typical symptom, and its presence can lead to misdiagnosis.

\section{Clinical cases}

\section{Case 1}

A 70-year-old patient was admitted to the emergency department in a serious general condition with clinical signs of hemorrhagic shock. According to relatives, the patient had repeatedly vomited bright red blood. A nasogastric tube was placed, from which bright red blood flew. He had regularly taken nonsteroidal anti-inflammatory drugs to relieve pain in the arm from an old fracture. Laboratory findings were as follows: Leukocytes 11.5x10\%/l, Hemoglobin - 56g/1, Er $2.9 \times 10^{9} / 1$, Platelets - $197 \times 10^{9} / 1$, prothrombin time was 73 seconds, INR -1.26 .

In an impaired general condition, the patient was admitted to the surgical ward for intensive care and blood transfusion. Once the hemodynamics was stabilized and the laboratory parameters were within normal range, fibrogastroscopy was performed.

Endoscopy revealed that the esophagus was of normal length and lumen. In the distal third - single, linear erosions with whitish beds from reflux of gastric juice through the cardia were seen. The cardia was passable. There was bright red blood in the stomach and a mass of coagulum in the lumen. Subcardially, on the anterior wall, a protruding blood vessel, slightly bleeding, was visualized during the examination. Endoscopic hemostasis was performed by combined therapy by injecting adrenaline solution around the blood vessel and applying four hemoclips. The bleeding was under control.

Further therapy included restoring water-salt balance, proton pump inhibitors, hemostatics, and nill per os diet. After seven days of bed rest, the patient was discharged in stable general health, with hemoglobin 103.

\section{Case 2}

An 85-year-old patient was admitted to the emergency department with complaints of vomiting, bloody stools, and black stools. He denied abdominal pain. A nasogastric tube is placed, from which hematin substances and a minimal amount of clear blood flew. He is constantly taking antihypertensive drugs.

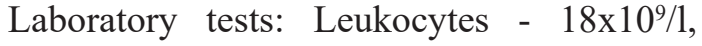
Hemoglobin - 87g/l, Er 3.0x10 $/ 1$, Platelets $357 \times 10^{9} / 1$, prothrombin time -92 , and INR $=1.06$. 
The stable hemodynamic status allowed fibrogastroscopy to be performed within 2 hours of admission. The esophagus had a normal length and lumen, and the cardia was passable. Hematin matter and a minimal amount of bright red blood in the lumen was seen. In the proximal third of the corpus, a protruding blood vessel, bleeding during the examination, was found along the greater curvature. Endoscopic hemostasis was performed by injecting adrenaline solution around the visibly protruding blood vessel, and a hemoclip was placed to control the bleeding.

The therapy continued with water-salt resuscitation saline infusion, blood transfusion, proton pump inhibitors, hemostatics, nill per os diet. After four days of hospital treatment, the patient was discharged in a stable general condition, with hemoglobin 98.

\section{Discussion}

Dieulafoy's lesion is a rare but important cause of non-varicose bleeding from the upper gastrointestinal tract. The risk of bleeding is highest in patients with concomitant pathology - cardiovascular disease, chronic kidney disease, hypertension, diabetes mellitus, and in patients taking nonsteroidal anti-inflammatory drugs (NSAIDs), anticoagulants or antiplatelet drugs [12]. Shin et al. (2014) investigated potential risk factors for bleeding in 42 patients [13]. The results showed that patients on antiplatelet agents are at a higher risk of bleeding than those taking NSAIDs. They found that chronic alcohol use was also a risk factor for bleeding.

The diagnosis is made by gastroscopy. Initial endoscopy detects the source of bleeding in 70\% of patients. Sometimes, several endoscopies are needed, with $6 \%$ of patients needing three or more to make a diagnosis. Reasons for a failed first endoscopy are the abundant amount of bright red blood in the stomach lumen, making it difficult to examine (44\%), or because the lesion is small, fine, and easily missed (56\%).

The characteristic endoscopic features of a Dieulafoy lesion are an isolated bulging blood vessel surrounded by a normal mucosa unrelated to an ulcer or an eroded large artery protruding into the lumen. If the lesion bleeds actively, clear blood is visualized, pulsing or leaking from the tip of the defect. In the absence of bleeding, a clot without an ulcer may be seen. The diagnostic criteria for Dieulafoy lesion are [14,15]:

- Active arterial bleeding or micropulsation from mucosal defect $<3 \mathrm{~mm}$ or thoroughly normal mucosa

- Visualization of a convex vessel, with or without bleeding, within a small mucosal defect or through a normal surrounding mucosa.

- The presence of a fresh clot tight-fitting to a small mucosal defect or to a normal-looking mucosa.

Endoscopic hemostasis is achieved in 90$100 \%$ of cases, although several endoscopies may be necessary for diagnostic and therapeutic purposes.

Treatment administered includes injection of adrenaline in a ratio of 1: 10000, application of hemoclips or ligation, plasma coagulation, monopolar or bipolar electrocoagulation [16]. Injection therapy is less effective, but in heavy bleeding, it can be easily applied at the bleeding site to control it, and then apply combined mechanical or thermal therapy for successful hemostasis [17]. Mechanical hemostasis has proved to be the safest and most effective method. Endoscopic hemoclips are more effective than stand-alone injection therapy [17]. Endoscopic band ligation (EBL) is an effective option when endoscopic hemoclip intervention is unsuccessful. There is a risk of ulcer formation in endoscopic variceal ligation EBL [18].

The most effective method of hemostasis is a combination of injection and mechanical therapy.

In case of unsuccessful endoscopic hemostasis, transcatheter angiographic embolization or surgical lesion is considered [19], according to the recommendations of the European Society of Gastrointestinal Endoscopy [20].

Recurrence of bleeding after initially successful endoscopic hemostasis is thought to be a decrease in Hemoglobin of $2 \mathrm{~g} / \mathrm{dL}$, the presence of visible signs of bleeding or the development of hemorrhagic shock after initial transfusion and stabilization. In these cases, repeated endoscopy is mandatory for a second attempt at hemostasis.

Despite effective endoscopic hemostasis, rebleeding occurs in $10 \%$ of patients, especially in the first 30 days after therapy [12]. The risk is higher with applying adrenaline alone, 
without combination with mechanical therapy or electrocautery [18].

\section{Conclusions}

Rare sources of upper gastrointestinal bleeding, such as Dieulafoy'slesion, should always be considered when performing gastroscopy for haematemesis and melena. The combination of injection therapy with Adrenaline solution and mechanical with the application of hemostatic clips helps achieve the best results for hemostasis of the Dieulafoy lesion so as to avoid surgery. In case of failure of endoscopic therapy, more invasive interventions such as transcatheter angiographic embolization and surgical treatment are considered.

\section{References}

1. Vlahova M, Lalev I, Sarafiloski I.Acute upper gastrointestinal tract bleeding as seen by a gastroenterologist-endoscopist. Health.bg journal. May 2016;1:20-3.

2. Chaer R, Helton WS. Dieulafoy's disease. AmColl Surg. 2003;196:290-6.

3. Marangoni G, Cresswell AB, Faraj W, et al. An uncommon cause of life-threatening gastrointestinal bleeding: 2 synchronous Dieulafoy lesions. J Paediatr Surg. 2009;44:441-3.

4. Prasad TR, Lim KW, Lim KT, et al. Bleeding jejunal Dieulafoy pseudo polyp: capsule endoscopic detection and laparoscopicassisted resection. J Laparoendosc Adv Surg Tech. 2007;17:509-12.

5. Alshumrani G, Almuaikeel M. Angiographic findings and endovascular embolization in Dieulafoy disease: a case report and literature review. DiagnInterventRadiol. 2006;12:1514.

6. Morowitz M, Markowitz R, Kamath $\mathrm{B}$, Allmen D. Dieulafoy's lesion segmental dilatation of the small bowel: an uncommon cause of gastrointestinal bleeding. J Paediatr Surg. 2004;39:1726-8.

7. Christoffersen RK, Nielsen TS, Vesterby A. Dieulafoy lesion of the esophagus causing massive upper gastrointestinal bleeding and death: a case report. Am J Forensic Med Pathol. 2012;33:186-7.

8. Baxter M, Aly EH. Ann R Dieulafoy's lesion: current trends in diagnosis and management. Coll Surg Engl. 2010;92:548-54.
9. Vlahova M. Acute upper gastrointestinal tract bleeding as seen by a gastroenterologistendoscopist. Monograph, Publishing Centre of Medical University - Pleven. 2020:34-58.

10. Scmulewitz N, Baillie J. Dieulafoy lesions; a review of 6 year's experience of a tertiary referral centre. Am J Gastrenterol. 2001;96:1689-94.

11. Clements J, Clements B, Loughrey M. Gastric Dieulafoy lesion: a rare cause of massive haematemesis in an elderly woman. BMJ Case Rep. 2018;bcr-2017.

12. Saleh R, Lucerna A, Espinosa J, Scali V. Dieulafoy lesion: the little known sleeping giant of gastrointestinal bleeds. Am J Emerg Med. 2016;34:2464.

13. Shin HJ, Ju JS, Kim KD, et al. Risk factors for Dieulafoy lesions in the upper gastrointestinal tract. Clin Endosc. 2015;48:228-33.

14. Chung IK, Kim EJ, Lee MS, Kim HS, Park $\mathrm{SH}$, et al. Bleeding Dieulafoy's lesions and the choice of endoscopic method: comparing the haemostatic efficacy of mechanical and injection methods. GastrointestEndosc. 2000;52:721-4.

15. Lee YT, Walmsley RS, Leong RW, Sung JJ. Dieulafoy's lesion.GastrointestEndosc. 2003;58:236-43.

16. Baldwin CL, Wilsey M. Pediatr. Three year old male with multiple Dieulafoy lesions treated with epinephrine injections via therapeutic endoscopy. Gastroenterol Hepatol Nutr. 2016; 19:276-280.

17. Nojkov B, Cappell MS. Distinctive aspects of peptic ulcer disease, Dieulafoy's lesion, and Mallory-Weiss syndrome in patients with advanced alcoholic liver disease or cirrhosis. World J Gastroenterol. 2016;22:446-66.

18. Park CH, Sohn YH, Lee WS, et al. The usefulness of endoscopic hemoclipping for bleeding Dieulafoy lesions. Endoscopy. 2003;35:388-92.

19. Saada M, Perek S, Agbaria M, Raz-Pasteur A. Massive gastrointestinal bleeding from a JejunalDieulafoy lesion: an extraordinary presentation. Case Rep Gastroenterol. 2019;13:508-13.

20. Gralnek IM, Dumonceau JM, Kuipers EJ et al. Diagnosis and management of nonvariceal upper gastrointestinal hemorrhage: European Society of Gastrointestinal Endoscopy (ESGE) Guideline. Endoscopy 2015;47:1-46. 